\title{
Digital Microfluidics for Cell-Based Screening
}

\author{
Aaron R. Wheeler,* Irena Barbulovic-Nad** and Samuel Au** \\ *Department of Chemistry, University of Toronto, 80 St. George Street, Toronto, Ontario, \\ M5S 3H6, Canada \\ **Institute of Biomaterials and Biomedical Engineering, University of Toronto, Rosebrugh \\ Building, 164 College Street, Room 407, Toronto, Ontario, M5S 3G9, Canada
}

Integrated robotic workstations have revolutionized drug discovery, enabling high-throughput screening (HTS) on an unprecedented scale. Such technology does not come cheap, howeverrobotic workstations are expensive, and the consumables costs (multiwell plates, pipette tips, chemicals, etc.) can make the running of even a single assay prohibitive. If these powerful methods are to be available to any but the most wealthy of laboratories, more efficient HTS technologies must be developed.

One such technology is microfluidics - several research groups are developing microchannelbased HTS methods, which are characterized by reduced reagent consumption, analysis time, and assay cost. While these strategies are promising, they are limited by the difficulty of controlling many different reagents simultaneously in interconnected channels, and by the requirement of specialized and dedicated detection systems. We are developing new screening assays using an alternative paradigm for fluid transport, called digital microfluidics (DMF). In DMF, fluid is manipulated as discrete droplets on an open platform, which makes simultaneous control of reagents straightforward and facile. Here, we present the first DMF methods for multiplexed apoptosis assays using arrays of droplets containing cultured cells, interfaced with conventional detection apparatus (i.e., a fluorescence multiwell plate reader). These methods, which were implemented in inexpensive devices with no moving parts, were characterized by $\sim 100 \mathrm{x}$ lower reagent consumption relative to conventional multiwell plate assays.

In addition to assays, we have developed DMF-driven methods for implementing all of the steps required for long-term mammalian cell culture - seeding, growing, passaging, and re-seeding. IN this work, mammalian cells are seeded in a DMF device and maintained in droplets of growth media for multiple days. They exhibit growth characteristics and morphology comparable to cells cultured in standard tissue culture flasks. Once the cells are confluent, they are passaged in using standard subculture protocols (washing, harvesting and splitting cells). The method is capable of passaging several generations of cells on the same device. These results suggest that DMF has great potential for use in automated cell culture as well as being an analytical tool for implementing cell-based assays on the microscale, perhaps in place of (or in addition to) the gold standard of robotic workstations. 